\title{
Evaluation of intratumoral heterogeneity by using diffusion kurtosis imaging and stretched exponential diffusion-weighted imaging in an orthotopic hepatocellular carcinoma xenograft model
}

\author{
Ran Guo ${ }^{1,2}$, Shuo-Hui Yang ${ }^{3 \#}$, Fang Lu ${ }^{3}$, Zhi-Hong Han ${ }^{4}, \mathrm{Xu} \mathrm{Yan}^{5}$, Cai-Xia Fu ${ }^{6}$, Meng-Long Zhao ${ }^{1,2}$, \\ Jiang Lin ${ }^{1,2 \#}$ \\ ${ }^{1}$ Department of Radiology, Zhongshan Hospital, Fudan University, Shanghai 200032, China; ${ }^{2}$ Shanghai Institute of Medical Imaging, Shanghai \\ 200032, China; ${ }^{3}$ Department of Radiology, ${ }^{4}$ Department of Pathology, Shuguang Hospital, Shanghai University of Traditional Chinese Medicine, \\ Shanghai 200021, China; ${ }^{5}$ MR Scientific Marketing, Siemens Healthcare, Shanghai 201318, China; ${ }^{6}$ Siemens Shenzhen Magnetic Resonance Ltd., \\ Shenzhen 518057, China
}

"These authors contributed equally to this work.

Correspondence to: Shuo-Hui Yang, MD, PhD. Department of Radiology, Shuguang Hospital, Shanghai University of Traditional Chinese Medicine, 185 Pu An Road, Shanghai, 200021, China. Email: caddie_yang1980@aliyun.com; Jiang Lin, MD, PhD. Department of Radiology, Zhongshan Hospital, Fudan University, 180 Fenglin Road, Shanghai 200032, China. Email: lin.jiang@zs-hospital.sh.cn.

Background: To investigate the value of diffusion kurtosis imaging (DKI) and diffusion-weighted imaging (DWI) with a stretched exponential model (SEM) in the evaluation of tumor heterogeneity in an orthotopic hepatocellular carcinoma (HCC) xenograft model.

Methods: Thirty orthotopic HCC xenograft nude mice models were established and randomly divided into two groups, the sorafenib induction group $(n=15)$ and control group $(n=15)$. Every mouse in each group underwent MRI with DKI and SEM on a 1.5T MR scanner at 7, 14, and 21 days after sorafenib intervention. DKI and SEM parameters including mean kurtosis (MK), mean diffusivity (MD), $\alpha$, and distributed diffusion coefficient (DDC) were measured, calculated, and compared between the two groups and among different time points. Sequential correlations between histopathological results including necrotic fraction (NF), micro-vessel density (MVD), Ki-67 index, standard deviation (SD), and kurtosis from hematoxylin-eosin staining, and DKI and SEM parameters were analyzed.

Results: MK, MD, and DDC of HCC in the sorafenib induction group were significantly higher than those in the control group at each time point $(\mathrm{P}<0.05)$, while $\alpha$ was significantly lower $(\mathrm{P}<0.05)$. Significantly positive correlations were found between $\mathrm{MK}$ and $\mathrm{NF}(\mathrm{r}=0.693, \mathrm{P}=0.010), \mathrm{SD}(\mathrm{r}=0.785, \mathrm{P}=0.003)$, kurtosis $(\mathrm{r}=0.779, \mathrm{P}=0.003)$, between $\mathrm{MD}$ and $\mathrm{NF}(\mathrm{r}=0.794, \mathrm{P}=0.003)$, SD ( $\mathrm{r}=0.629, \mathrm{P}=0.020)$, kurtosis ( $\mathrm{r}=0.645$, $\mathrm{P}=0.018)$, and between DDC and NF ( $\mathrm{r}=0.800, \mathrm{P}=0.003)$, SD $(\mathrm{r}=0.636, \mathrm{P}=0.020)$, kurtosis $(\mathrm{r}=0.664$, $\mathrm{P}=0.016)$, and significantly negative correlations were observed between $\alpha$ and $\mathrm{NF}(\mathrm{r}=-0.704, \mathrm{P}=0.009)$, SD $(\mathrm{r}=-0.754, \mathrm{P}=0.003)$, and kurtosis $(\mathrm{r}=-0.792, \mathrm{P}=0.003)$ in the sorafenib induction group.

Conclusions: DKI and SEM parameters may be potentially useful for evaluating intratumoral heterogeneity in HCC.

Keywords: Tumor heterogeneity; magnetic resonance imaging; diffusion kurtosis imaging (DKI); stretched exponential model (SEM); hepatocellular carcinoma (HCC)

Submitted Mar 20, 2019. Accepted for publication Aug 21, 2019.

doi: $10.21037 /$ qims.2019.08.18

View this article at: http://dx.doi.org/10.21037/qims.2019.08.18 


\section{Introduction}

Heterogeneity, arising from the genetic instability of tumors, leads to an apparently chaotic growth of tumor structures including high cellularity, necrosis, immature angiogenesis, hemorrhage, and myxoid change, etc. (1). It poses a critical challenge for clinicians to accurately predict the physiological behavior and clinical outcome of a tumor (2). Intratumoral heterogeneity is associated with tumor development, progression, and response to treatment (3).

Diffusion-weighted imaging (DWI) can be utilized to assess tumor cell proliferation, and tissue necrosis (4). However, the conventional mono-exponential diffusion model is not suitable for evaluating tumor heterogeneity because of the non-Gaussian behavior of water in the tumor (5). This non-Gaussian behavior of diffusion which reflects tissue heterogeneity and irregularity, however, can be explored by using DWI with high b values and advanced modeling of DWI data $(6,7)$. Recently, several studies have reported that diffusion kurtosis imaging (DKI) and stretched exponential model (SEM) derived from DWI can provide valuable information on tumor microstructural complexity, which could be used for improved characterization of tumors, evaluation of prognosis, and monitoring of their therapeutic efficacy $(5,8-10)$.

Hepatocellular carcinoma (HCC) is one of the most frequently diagnosed cancers worldwide (11). Despite endeavors to improve prognosis, the overall survival rate of HCC is still rather dismal since it is a heterogeneous disease with wide variations in outcomes and response to therapy (12). Although a few studies have tentatively investigated the values of DKI and SEM in demonstration of the heterogeneity of HCC (13-15), a directly quantitative correlation between DKI and SEM parameters with the histopathological results of HCC heterogeneity, is scarce.

Sorafenib has antiangiogenic and antiproliferative effects on HCC (16). Using a low dose of $10 \mathrm{mg} / \mathrm{kg}$ body weight, a prior study reported that sorafenib could induce tumor ischemia, hypoxia, and necrosis, and partially inhibit tumor proliferation and angiogenesis (16). As a result, a low dose of sorafenib could presumably more rapidly induce a heterogeneous HCC compared with its natural growth status. Hence, using a sorafenib-induced HCC xenograft animal model, we aimed to sequentially correlate quantitative DWI parameters from DKI and SEM with quantitative histopathologic tumor tissue composition for the purpose of evaluating HCC heterogeneity.

\section{Methods}

\section{Orthotopic HCC xenograft animal model and sorafenib induction}

This experiment was approved by the Institutional Animal Care and Use Committee of our hospital. The human HCC-LM3 cell line and 4-6-week-old male BALB/c nude mice (Shanghai Institute of Materia Medica, Chinese Academy of Sciences, Shanghai, China) weighing between 23 and $25 \mathrm{~g}$ were used to establish the orthotopic HCC xenograft model (4). Briefly, HCC-LM3 cells $\left(5 \times 10^{6} / 0.2 \mathrm{~mL} / \mathrm{site}\right)$ were inoculated subcutaneously in the left axilla of a nude mouse. When HCC tumor grew to $1 \mathrm{~cm}$ in diameter, it was removed and cut into the volume of $1 \mathrm{~mm}^{3}$ blocks. A piece of tumor block was implanted into the left lobe of the liver of the nude mouse. At the $14^{\text {th }}$ day after implantation, the mice were randomly divided into the sorafenib induction group $(n=15)$ and control group $(n=15)$. The mice in the induction group were administered daily with sorafenib solution at a dose of $10 \mathrm{mg} / \mathrm{kg}$ body weight by oral gavage (16). The mice in the control group were administered with $0.2 \mathrm{~mL}$ of $0.9 \%$ saline once a day. At the $7^{\text {th }}, 14^{\text {th }}$, and $21^{\text {st }}$ day after the intervention, 5 mice were taken randomly from the induction group and control group for MRI examinations and the following histopathological correlations.

\section{MRI techniques}

All HCC nude mice models were examined using a $1.5 \mathrm{~T}$ MRI scanner (MAGNETOM Aera; Siemens Healthcare, Erlangen, Germany) with a 16-channel wrist coil. All mice were anesthetized with $1.5 \%$ sodium pentobarbital at a dose of $40 \mathrm{mg} / \mathrm{kg}$ body weight by intraperitoneal injection. The mouse was then placed in the prone position in an animal cradle with a belt holding the abdomen to limit respiratory motion.

Coronal and transverse T2-weighted imaging (T2WI) and transverse T1-weighted imaging (T1WI) were done using turbo spin echo with a section thickness of $2 \mathrm{~mm}$, an intersection gap of $0.2 \mathrm{~mm}$, and a field of view (FOV) of $100 \times 100 \mathrm{~mm}^{2}$. Other parameters of coronal T2WI sequence were as follows: repetition time $(\mathrm{TR})=4,000.0 \mathrm{~ms}$, echo time $(\mathrm{TE})=74.0 \mathrm{~ms}$, bandwidth $=150.0 \mathrm{~Hz} /$ pixel, flip angle $(\mathrm{FA})=150^{\circ}$, matrix size $=272 \times 320$, acquisition time $=$ 1 minute 48 s. Other parameters of transverse T1WI were 


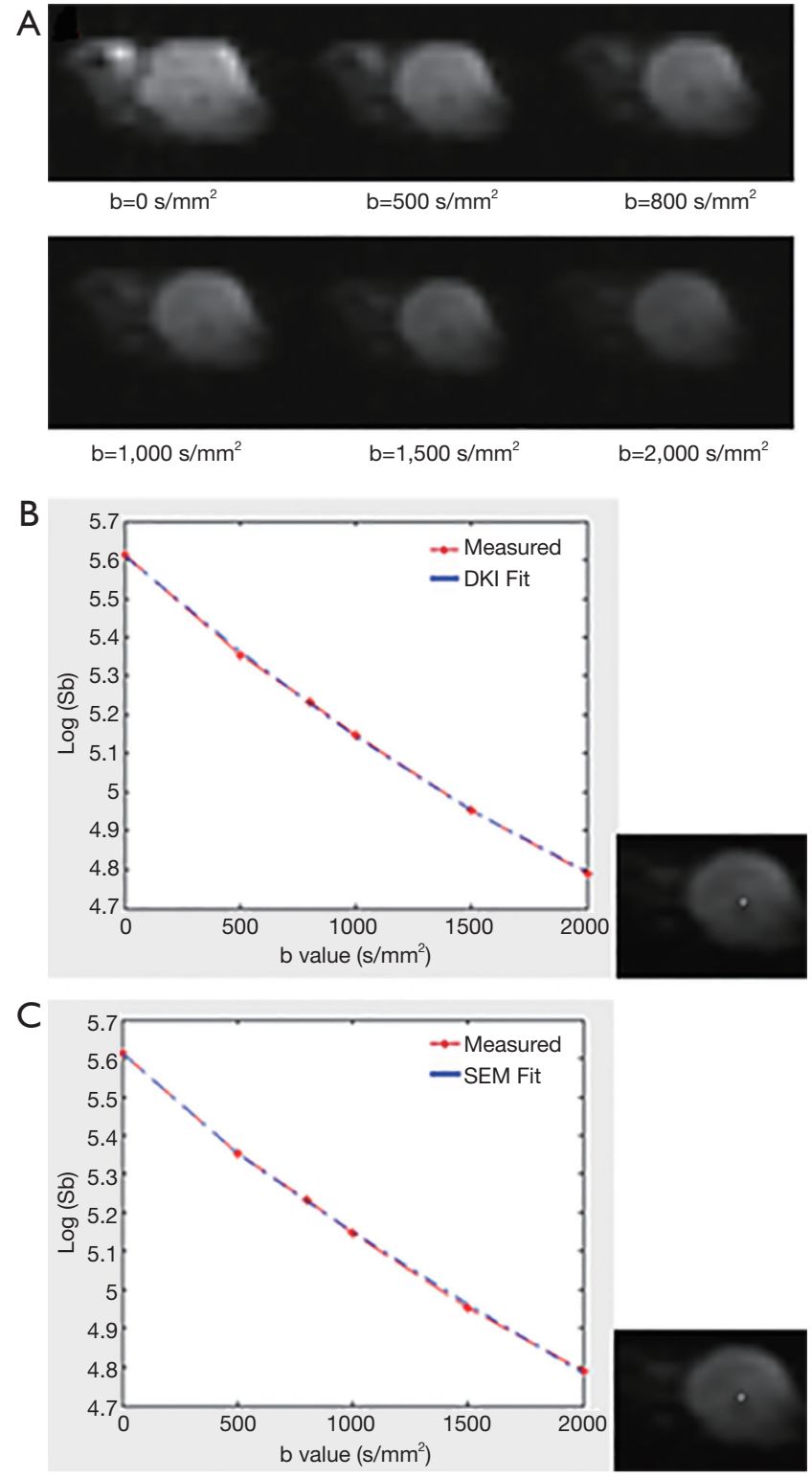

Figure 1 DWI images and the pixel-based fitted curves of DKI and SEM of a hepatocellular carcinoma nude mouse in the control group on the $21^{\text {st }}$ day. (A) DWI images ( $b=0,500,800,1,000,1,500$ and 2,000 s/mm ${ }^{2}$. (B,C) The pixel-based fitted curves of DKI and SEM. DWI, diffusion-weighted imaging; DKI, diffusion kurtosis imaging; SEM, stretched exponential model.

as follows: $\mathrm{TR}=480.0 \mathrm{~ms}$, TE $=13.0 \mathrm{~ms}$, bandwidth $=$ $130.0 \mathrm{~Hz} /$ pixel, $\mathrm{FA}=90^{\circ}$, matrix size $=410 \times 512$, acquisition time $=1$ minute $25 \mathrm{~s}$. Parameters of transverse T2WI were as follows: $\mathrm{TR}=4,000.0 \mathrm{~ms}, \mathrm{TE}=103.0 \mathrm{~ms}$, bandwidth $=$ 150.0 Hz/pixel, $\mathrm{FA}=150^{\circ}$, matrix size $=272 \times 320$, acquisition time $=1$ minute 48 s. For DKI-SEM measurement, a freebreathing single-shot echo planar DWI sequence was applied in the transverse plane using three orthogonal diffusion directions with $6 \mathrm{~b}$ values [average number] of 0 [1], 500 [2], 800 [3], 1,000 [4], 1,500 [6], and 2,000 [6] s/mm² $(7,13)$. Parameters of DKI-SEM sequences were as follows: section thickness $=2 \mathrm{~mm}$, intersection gap $=0.4 \mathrm{~mm}, \mathrm{FOV}$ $=176 \times 295 \mathrm{~mm}^{2}$, matrix size $=118 \times 198, \mathrm{TR}=5,000.0 \mathrm{~ms}$, TE $=75.0 \mathrm{~ms}$, bandwidth $=1,405 \mathrm{~Hz} /$ pixel. Parallel imaging with an acceleration factor of 2 was used to shorten the scan time and reduce image distortion.

\section{Image postprocessing and quantitative measurement}

The original DWI images were imported to an in-house developed postprocessing program based on MATLAB (Mathworks, Natick, MA, USA) for offline DKI and SEM calculation, which was described in a previous study (17). The noise of the diffusion images was first suppressed by applying a Gaussian filter with a full width at half maximum of $3 \mathrm{~mm}$. A voxel-by-voxel fitting was then performed by using the following equations, from which metrics of DKI and SEM were derived: $S(b) / S(0)=\exp$ $\left(-\mathrm{b} \cdot M D+1 / 6 \cdot \mathrm{b}^{2} \cdot M D^{2} \cdot M K\right) ; \mathrm{S}(\mathrm{b}) / \mathrm{S}(0)=\exp \left[-(\mathrm{b} \cdot D D C)^{\alpha}\right](5)$. $\mathrm{S}(\mathrm{b})$ is the signal intensity at a particular b value, and $\mathrm{S}(0)$ is the signal intensity without diffusion weighting. $M D$ is mean diffusivity, a corrected apparent diffusion coefficient (ADC) without non-Gaussian bias. MK is mean kurtosis and quantifies the deviation of water moving from the Gaussian distribution. DDC represents distributed diffusion coefficient reflecting the average rate of diffusion. The heterogeneity index $\alpha$, with a range of $0-1$, describes the heterogeneity of water diffusion (5). ADC of a monoexponential model is automatically generated by the postprocessing software. The pixel-based fitted curve was also drawn by using this software (Figure 1).

Diffusion images were analyzed and measured by two independent radiologists with 12- and 25-year experience in liver MR image interpretation. Both of them were blinded to the grouping of mice and the histopathological results. Tumor size defined by the greatest dimension on the transverse T2W images was measured (4). Both transverse T1W and T2W images were reviewed to exclude hemorrhage in the tumor. If there was hemorrhage found on this greatest dimension slice, the most adjacent slice would be analyzed. Regions of interest (ROIs) were manually drawn along the tumor border on the slice showing the greatest dimension of the tumor on ADC images $(4,17)$. Then, the ROIs were automatically 

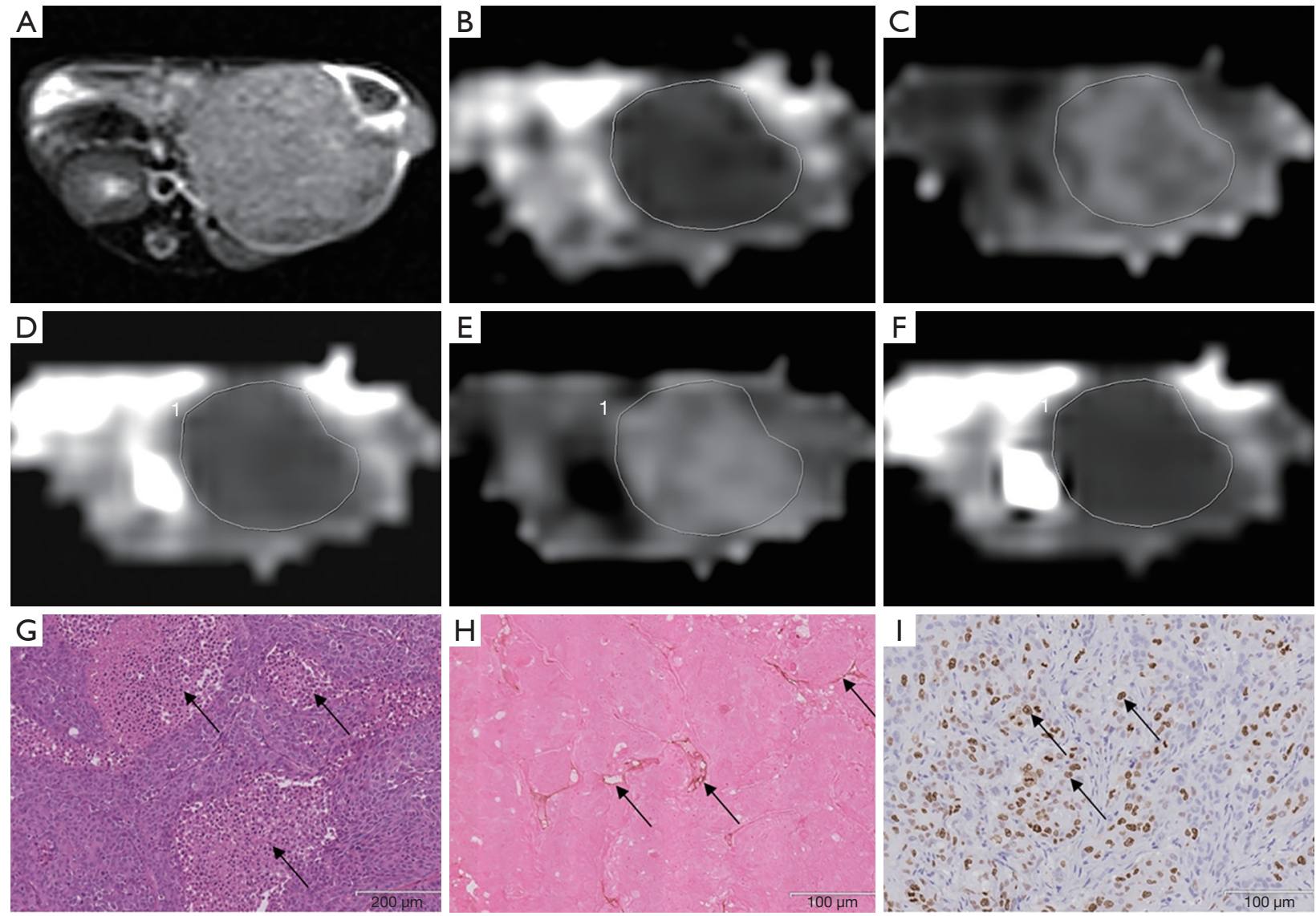

Figure 2 DKI and SEM images and corresponding histopathological images of a hepatocellular carcinoma in the sorafenib induction group on the $21^{\text {st }}$ day. (A) Transverse T2WI shows the greatest dimension of the tumor. (B) ADC map for ROI outlining the tumor. (C) MK map (MK =1.320). (D) MD map (MD =0.761 $\left.\times 10^{-3} \mathrm{~mm}^{2} / \mathrm{s}\right)$. (E) $\alpha$ map $(\alpha=0.735)$. (F) DDC map (DDC =0.602 $\left.\times 10^{-3} \mathrm{~mm}^{2} / \mathrm{s}\right)$. (G) Hematoxylin-eosin staining image shows patchy and irregular necrosis (black arrows, 10x) in the tumor. $(\mathrm{H})$ Anti-CD31 immunohistochemistry image shows sparsely intratumoral micro-vessels (black arrows, 20×). (I) Anti-Ki-67 immunohistochemistry image shows heterogeneously stained Ki-67 positive cells (black arrows, 20×). DKI, diffusion kurtosis imaging; SEM, stretched exponential model; T2WI, T2-weighted imaging; ADC, apparent diffusion coefficient; ROI, region of interest; MK, mean kurtosis; MD, mean diffusivity; DDC, distributed diffusion coefficient.

copied onto the DKI and SEM maps by the postprocessing software (Figure 2). Each observer measured the tumor twice on the same tumor slice, and the results were averaged. After a 4-week interval, these measurements were repeated to minimize any learning bias (18). The observers were aware of the goal of the study but were unaware of other information regarding the group and the time point of each model and the histopathological results. The final averaged values from the observer with 25 years of experience were used for subsequent statistical analysis.

\section{Histopathological analysis}

All nude mice were sacrificed after MRI examinations, and the tumors were removed and fixed in the $10 \%$ buffered formalin solution for at least 24 hours. With reference to the slice thickness of DKI-SEM sequences, each tumor was cut into the section of $2 \mathrm{~mm}$ thickness in its greatest dimensional transverse plane. The section was embedded in paraffin and then cut into a slice with a thickness of $3 \mu \mathrm{m}$.

Aperio ScanScope and Leica SCN400 (Leica biosystems, Buffalo Grove, IL, USA) were used to perform the scanning of all the histopathological slices including hematoxylin-eosin (HE), anti-CD31, and anti-Ki-67 by a pathologist with 10 years of experience in histopathological analysis. The scanning images of the whole slices were imported to a PC with a screen resolution of $1,377 \times 768$ pixels and post-processed with SlidePath 
Gateway Client software (Leica biosystems, Buffalo, IL, USA) for magnification, observation, and final image capturing. The histopathological measurements were performed on the captured images by using ImageJ software (v.1.48; National Institutes of Health, Bethesda, MD, USA) and Image-Pro Plus 6.0 software (Media Cybernetics, Rockville, MD, USA).

$\mathrm{HE}$ staining was performed to demonstrate the morphology and structure of HCC cells and mesenchymal tissues. The histogram analysis completed on the whole slice of the HE staining image with ImageJ software to obtain the parameters that reflect tumor heterogeneity: standard deviation (SD) and kurtosis. An ROI was manually drawn along the tumor border on this HE slice after conversion from color to grayscale (Figure 3) (19-23).

The measurement of tumor necrotic fraction (NF) was described in detail by a previous report (24). Briefly, five areas with four in each corner and one in the center on each HE whole slice were selected at lower power $(4 x$ and $10 \times$ magnification). Then, necrotic areas were obtained with Image-Pro Plus 6.0 in three higher-power FOVs (20x magnification) in each of the aforementioned five areas. Finally, NF was calculated according to the following equation: $\mathrm{NF}=$ Area $_{\text {necrosis in } \mathrm{FOV}} / \mathrm{Area}_{\text {tumor in } \mathrm{FOV}}$.

Anti-CD 31 staining (Abcam, Cambridge, UK) was performed to obtain the micro-vessel density (MVD). Three regions with the densest CD 31-positive vessels of anti-CD 31 staining images were found at $4 \times$ magnification. These regions were magnified by $20 \times$, and then these corresponding images were captured (4). MVD was obtained on these captured images with ImageJ software.

Anti-Ki-67 staining (Abcam, Cambridge, UK) was done to show the cell proliferation of the tumor. Ki-67 index was obtained similarly according to a previous report (25).

Every aforementioned histopathological index was measured twice, and the results were averaged for further analysis.

\section{Statistical analysis}

Mann-Whitney $U$-test was performed to analyze the differences between DKI and SEM parameters along with the differences of histopathological results between the induction and control groups at each time point. Differences in DKI and SEM parameters among different time points in both the induction and control groups were evaluated by using the Kruskal-Wallis $H$-test and Mann-Whitney $U$-test. The Spearman rank correlation test was used to investigate the correlations between DKI and SEM parameters and histopathological results, and the Benjamini-Hochberg method was performed to control for a false discovery rate. The correlation coefficient rho $(r)$ was obtained to evaluate the degree of correlations: $0 \leq|r|<0.2$ represented a poor or no relationship; $0.2 \leq|r| \leq 0.4$ was weak; $0.4<|r| \leq 0.6$ was moderate; $0.6<|r| \leq 0.8$ was good; $|r|>0.8$ was excellent (4). Two-way analysis of variance (ANOVA) was performed to analyze the effects of three-time points and the corresponding accumulated effects of sorafenib on DKI and SEM parameters. All the intra- and inter-observer agreement of DKI and SEM parameters and intra-observer agreement of histopathological measurements were evaluated by using intraclass correlation coefficients (ICCs) in the induction group. The inter-observer agreement was defined as the agreement between the two observers with the averaged values from two measurements at a 4-week interval. ICCs were defined as follows: $\leq 0.40$, poor to fair reliability; $0.41-0.60$, moderate reliability; $0.61-0.80$, good reliability; $>0.80$, excellent reliability (26). Measurement data are expressed as (mean $\pm \mathrm{SD}$ ). $\mathrm{P}<0.05$ was considered statistically significant. Statistical analysis was performed using the SPSS software (v.18.0; IBM, Armonk, NY, USA).

\section{Results}

The DKI-SEM acquisition and analysis were successfully performed in all 30 mice, and all the tumors were clearly displayed on diffusion images. Intratumoral hemorrhage was observed in two mice in the induction group (one at the $14^{\text {th }}$ day and one at the $21^{\text {st }}$ day after sorafenib intervention) and in one mouse in the control group (at the $21^{\text {st }}$ day after saline administration), but none of them were found on the measuring slice of the tumor.

\section{Comparisons of DKI and SEM parameters between the sorafenib induction group and control group}

MK, MD, and DDC of HCC in the sorafenib induction group were significantly higher than those in the control group at each time point, whereas $\alpha$ was significantly lower (Table 1, Figure 4).

Comparisons of DKI and SEM parameters at different time points in the sorafenib induction group and control group

Kruskal-Wallis $H$-test showed that DKI and SEM 

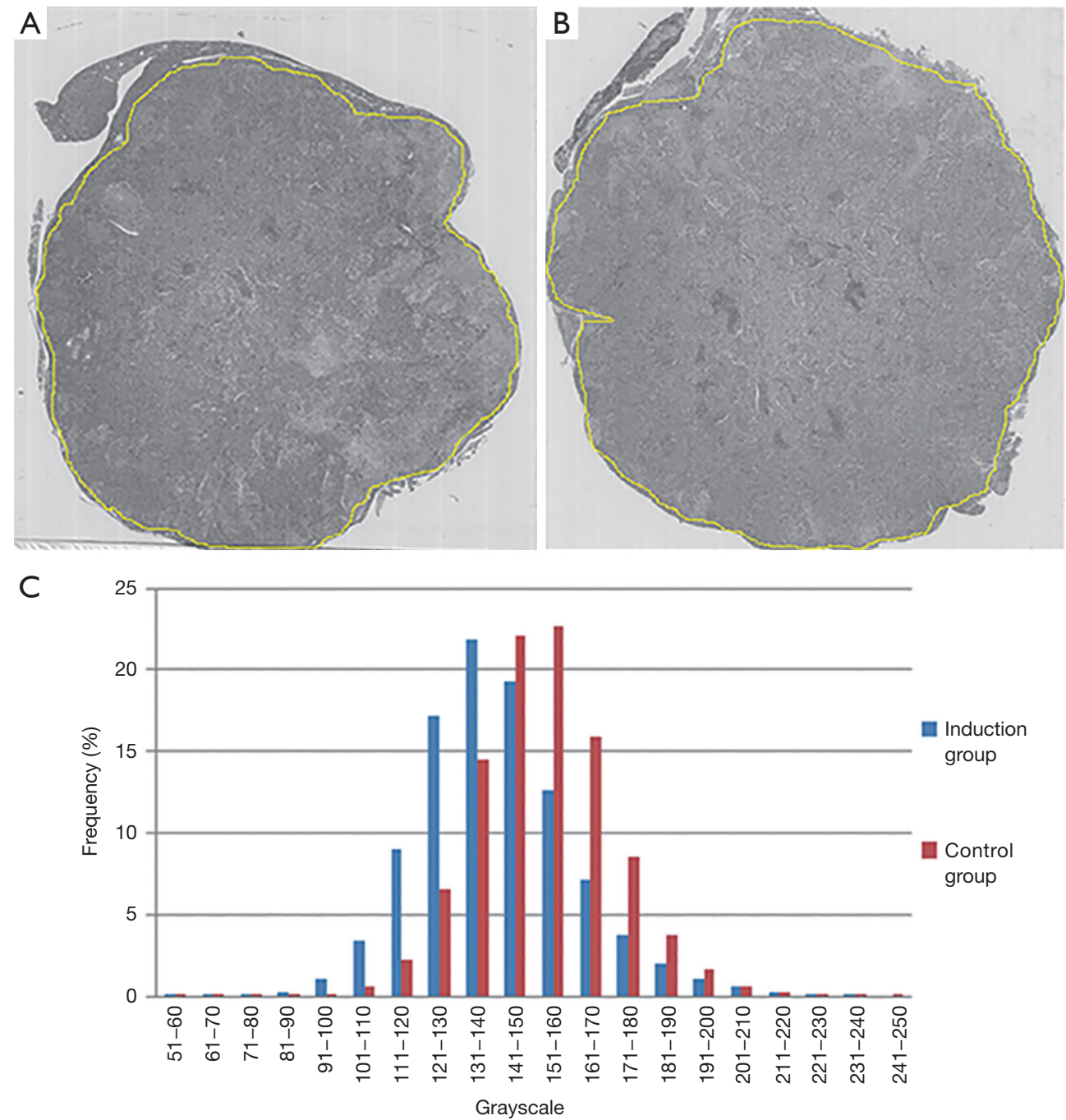

Figure 3 Grayscale images from two whole slice hematoxylin-eosin staining HCCs and their frequency histograms of the pixel distribution on the $21^{\text {st }}$ day after intervention. (A) Grayscale image of a tumor in the sorafenib induction group ( $\mathrm{SD}=20.645$, kurtosis $\left.=5.451\right)$; (B) grayscale image of a tumor in the control group ( $\mathrm{SD}=16.161$, kurtosis $=2.850)$; $(\mathrm{C})$ frequency histograms of the pixel distribution in the grayscale images of the tumors in sorafenib induction group (blue) and control group (red). HCC, hepatocellular carcinoma; SD, standard deviation.

parameters of the induction group at different time points were significantly different $\left(\mathrm{MK}: \chi^{2}=12.150, \mathrm{P}=0.002\right.$; MD: $\chi^{2}=11.786, \mathrm{P}=0.003 ; \alpha: \chi^{2}=11.180, \mathrm{P}=0.004$; DDC: $\left.\chi^{2}=12.020, \mathrm{P}=0.002\right)$. In the induction group, $\mathrm{MK}, \mathrm{MD}$, and DDC were significantly higher on the $14^{\text {th }}$ and $21^{\text {st }}$ days than those on the $7^{\text {th }}$ day $(M K: Z=-2.440, P=0.015$ and $\mathrm{Z}=-2.627, \mathrm{P}=0.009 ; \mathrm{MD}: \mathrm{Z}=-2.402, \mathrm{P}=0.016$ and $\mathrm{Z}=-2.611$, $\mathrm{P}=0.009$; DDC: $\mathrm{Z}=-2.402, \mathrm{P}=0.016$ and $\mathrm{Z}=-2.611$, $\mathrm{P}=0.009)$. Significant increases of these parameters were also found on the $21^{\text {st }}$ day $v$ s. $14^{\text {th }}$ day (MK: $Z=-2.627$, $\mathrm{P}=0.009$; $\mathrm{MD}: \mathrm{Z}=-2.514, \mathrm{P}=0.012$; DDC: $Z=-2.611$,
$\mathrm{P}=0.009)$. Meanwhile, $\alpha$ was significantly lower on the $14^{\text {th }}$ and $21^{\text {st }}$ days compared with the $7^{\text {th }}$ day $(\mathrm{Z}=-2.207, \mathrm{P}=0.027$ and $Z=-2.627, P=0.009)$, and lower on the $21^{\text {st }}$ day when compared with the $14^{\text {th }}$ day $(\mathrm{Z}=-2.440, \mathrm{P}=0.015)$ (Figure 4). In the control group, $M K$ increased slightly on the $14^{\text {th }}$ and $21^{\text {st }}$ days, while $\alpha$ displayed a decreasing trend from the $7^{\text {th }}$ to the $21^{\text {st }}$ day $(\mathrm{P}>0.05)$. MD and DDC declined slightly on the $14^{\text {th }}$ day, and then increased on the $21^{\text {st }}$ day $(\mathrm{P}>0.05)$. No significant differences were found in any of these DKI and SEM parameters at three time points in the control group $\left(M K: \chi^{2}=4.973, \mathrm{P}=0.083 ; M D: \chi^{2}=4.340, \mathrm{P}=0.114 ; \alpha\right.$ : 
Table 1 Comparisons of DKI and SEM parameters of HCC between the sorafenib induction group and control group

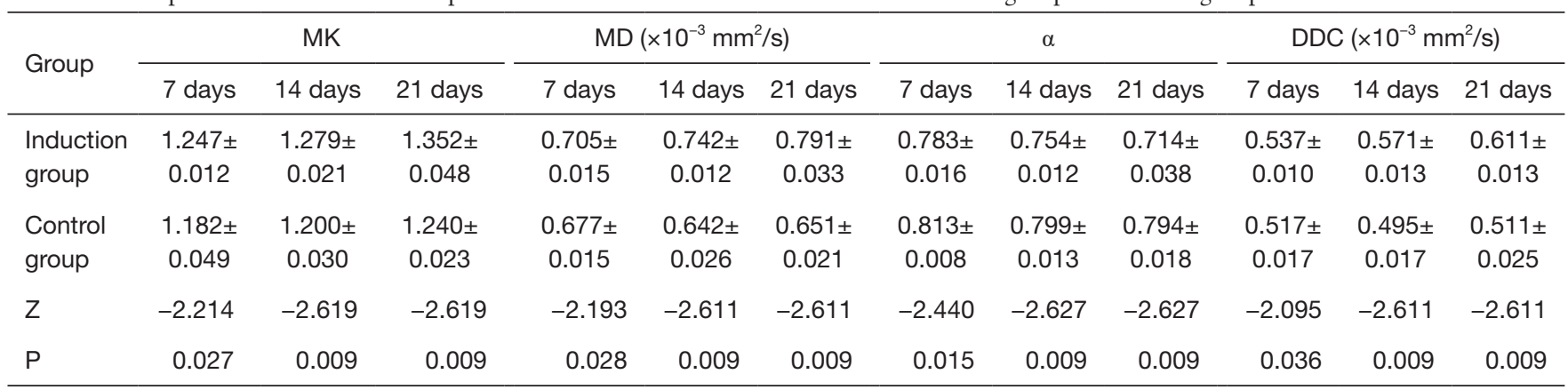

DKI, diffusion kurtosis imaging; SEM, stretched exponential model; HCC, hepatocellular carcinoma; MK, mean kurtosis; MD, mean diffusivity; DDC, distributed diffusion coefficient

A

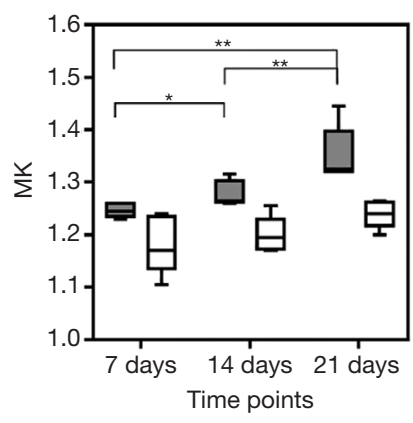

C

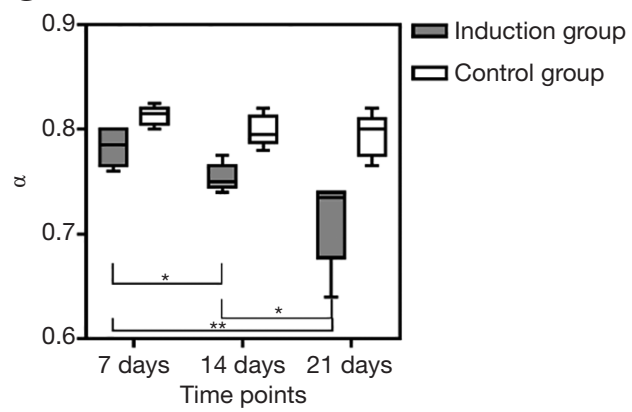

B

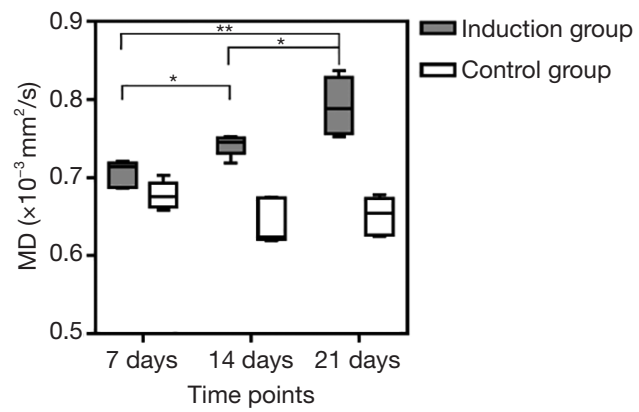

$\mathrm{D}$

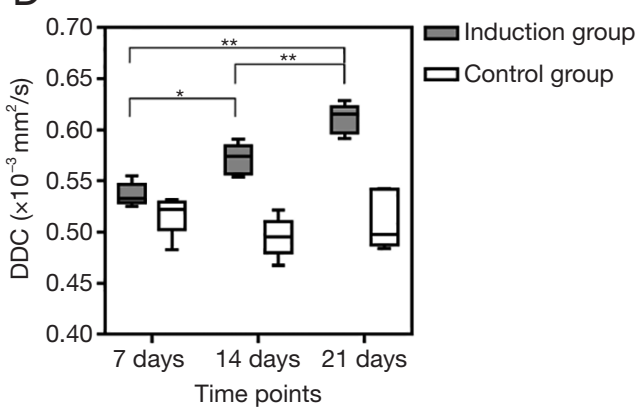

Figure 4 Sequential comparisons of DKI and SEM parameters of HCC in the sorafenib induction group and control group at different time points. Box-plots show sequential changes of MK (A), MD (B), $\alpha(C)$, and DDC (D) in these two groups. All parameters were tested with Mann-Whitney $U$-test $\left({ }^{*} \mathrm{P}<0.05,{ }^{* *} \mathrm{P}<0.01\right)$. Center line $=$ median; upper and lower margins of box $=$ upper and lower quartiles. DKI, diffusion kurtosis imaging; SEM, stretched exponential model; HCC, hepatocellular carcinoma; MK, mean kurtosis; MD, mean diffusivity; DDC, distributed diffusion coefficient.

$\left.\chi^{2}=3.438, \mathrm{P}=0.179 ; \mathrm{DDC}: \chi^{2}=1.758, \mathrm{P}=0.415\right)$.

\section{Comparisons of tumor size and histogram parameters in hematoxylin-eosin staining between the sorafenib induction group and control group}

At each time point, the tumor size in the induction group was slightly smaller than that in the control group with no significant difference observed $\left(7^{\text {th }}\right.$ day: $1.220 \pm 0.264$ vs. $1.240 \pm 0.136 \mathrm{~cm}, \mathrm{P}=0.750 ; 14^{\text {th }}$ day: $1.640 \pm 0.215$ vs. $1.660 \pm 0.080 \mathrm{~cm}, \mathrm{P}=0.595 ; 21^{\text {st }}$ day: $1.680 \pm 0.117$ vs. $1.940 \pm 0.301 \mathrm{~cm}, \mathrm{P}=0.240)$. SD and kurtosis of histogram parameters in the induction group were significantly higher than those in the control group $(\mathrm{P}<0.05)$ (Table 2, Figure 3). 
Table 2 Comparisons of SD and kurtosis of HCC in hematoxylin-eosin staining between the sorafenib induction group and control group

\begin{tabular}{|c|c|c|c|c|c|c|}
\hline Group & \multicolumn{3}{|c|}{ SD } & \multicolumn{3}{|c|}{ Kurtosis } \\
\hline Induction group & $16.903 \pm 0.531$ & $18.727 \pm 1.500$ & $20.449 \pm 0.421$ & $-4.890 \pm 2.259$ & $-1.070 \pm 1.301$ & $6.250 \pm 1.718$ \\
\hline Control group & $15.804 \pm 0.699$ & $16.153 \pm 0.758$ & $17.119 \pm 1.110$ & $-9.346 \pm 2.158$ & $-6.256 \pm 2.366$ & $-1.350 \pm 4.487$ \\
\hline Z & -2.193 & -2.402 & -2.611 & -2.193 & -2.611 & -2.611 \\
\hline
\end{tabular}

SD, standard deviation; HCC, hepatocellular carcinoma

\section{Comparisons of histopathological results (NF, MVD and Ki-67 index) between the sorafenib induction group and control group}

At each time point, NF of the induction group was significantly greater than that of the control group, and significantly lower MVD and Ki-67 index were observed in the induction group than those in the control group $(\mathrm{P}<0.05)$ (Table 3).

\section{Correlations between DKI and SEM parameters and histopathological results in the sorafenib induction group and control group}

In the induction group, MK, MD, and DDC showed significantly positive correlations with NF, SD, and kurtosis. $\alpha$ was significantly and negatively correlated with NF, SD, and kurtosis. No correlations were found between DKI and SEM parameters and MVD and Ki-67 index (Table 4). In the control group, there was no correlation between DKI and SEM parameters and NF, MVD, and Ki-67 index, and $\mathrm{SD}$ and kurtosis (Table 5).

\section{Comparisons of the effects of three time points and the} corresponding accumulated effects of sorafenib on DKI and SEM parameters in the HCC models

Two-way ANOVA revealed significantly accumulated effects of sorafenib from 7 to 21 days on all DKI and SEM parameters (MK: $\mathrm{F}=13.713, \mathrm{P}<0.001 ; \mathrm{MD}: \mathrm{F}=43.904$, $\mathrm{P}<0.001 ; \alpha: \mathrm{F}=15.426, \mathrm{P}<0.001 ;$ and DDC: $\mathrm{F}=38.050$, $\mathrm{P}<0.001)$. However, no significant effects from these three time points on DKI and SEM parameters were observed (MK: $\mathrm{F}=3.151, \mathrm{P}=0.061 ; \mathrm{MD}: \mathrm{F}=2.820, \mathrm{P}=0.079 ; \alpha$ : $\mathrm{F}=0.963, \mathrm{P}=0.396$ and DDC: $\mathrm{F}=1.840, \mathrm{P}=0.180$ ).

\section{Intra- and interobserver agreement on DKI and SEM parameters in the sorafenib induction group}

The intra- and interobserver agreements for measuring all DKI and SEM parameters were excellent (Table 6).

The intra-observer agreement on histopathologic SD, kurtosis, NF, MVD, and Ki-67 index were 0.979 [95\% confidence interval (CI): 0.941-0.993], 0.976 (95\% CI: 0.932-0.992), 0.973 (95\% CI: 0.922-0.991), 0.923 (95\% CI: $0.790-0.974)$, and 0.965 (95\% CI: 0.898-0.988) $(\mathrm{P}<0.001)$, respectively.

\section{Discussion}

The heterogeneity of a tumor is related to the presence and changes of different components within the tumor during its formation, progression, and treatment $(3,27,28)$. Patients with higher heterogeneity are likely to have a poorer prognosis, which may be secondary to more aggressive biology or treatment resistance $(3,29)$. Prior knowledge of tumor heterogeneity, especially in a non-invasive way, is critical both before and after treatment. This study demonstrated that parameters from DKI and SEM could largely reflect the intratumoral heterogeneity of HCC. We also observed that the tumor heterogeneity changed over time, which was correlated with sequentially obtained histopathological results. Therefore, DKI and SEM parameters may be used as valuable imaging biomarkers for evaluating the heterogeneity in HCC.

In this experiment, at each time point after $10 \mathrm{mg} / \mathrm{kg}$ sorafenib intervention, NF in the induction group was significantly larger than that in the control group, while MVD and Ki-67 index was significantly lower. These results indicate that $10 \mathrm{mg} / \mathrm{kg}$ sorafenib did have destructive effects on HCC. We further found that SD and kurtosis 


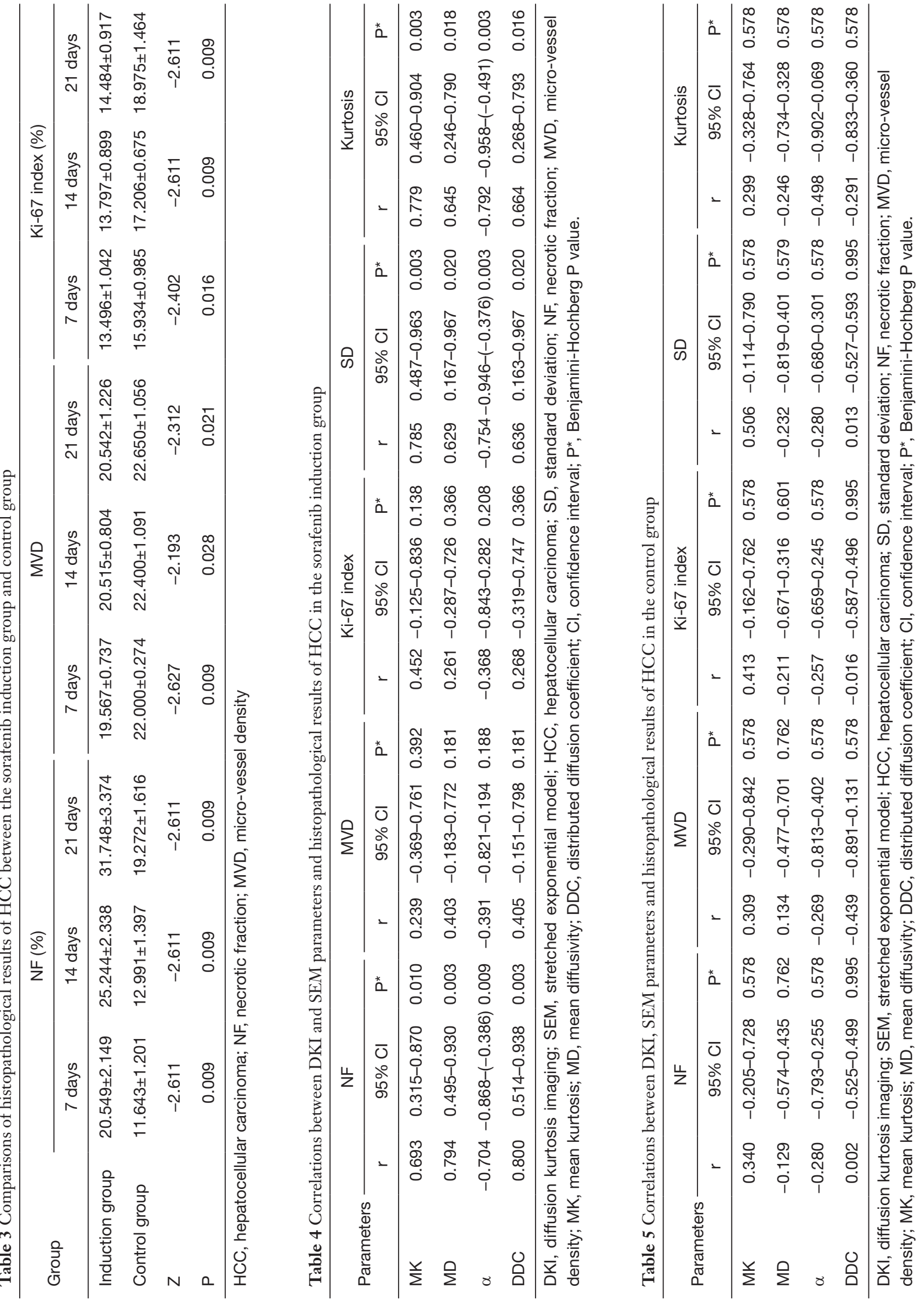


Table 6 Intra- and inter-observer agreement on DKI and SEM parameters in the sorafenib induction group

\begin{tabular}{|c|c|c|c|c|c|c|c|c|c|}
\hline Parameters & \multicolumn{3}{|c|}{ Reader $1^{*}$} & \multicolumn{3}{|c|}{ Reader 2} & \multicolumn{3}{|c|}{ Inter-observer } \\
\hline MK & 0.920 & $0.625-0.977$ & $<0.001$ & 0.942 & $0.725-0.983$ & $<0.001$ & 0.887 & $0.495-0.967$ & $<0.001$ \\
\hline MD & 0.958 & $0.882-0.986$ & $<0.001$ & 0.932 & $0.811-0.977$ & $<0.001$ & 0.949 & $0.855-0.982$ & $<0.001$ \\
\hline$\alpha$ & 0.938 & $0.831-0.979$ & $<0.001$ & 0.910 & $0.758-0.969$ & $<0.001$ & 0.880 & $0.680-0.958$ & $<0.001$ \\
\hline
\end{tabular}

${ }^{*}$ Reader 1: a radiologist with 25-year experience in liver MR image interpretation. DKI, diffusion kurtosis imaging; SEM, stretched exponential model; MK, mean kurtosis; MD, mean diffusivity; DDC, distributed diffusion coefficient; ICCs, intraclass correlation coefficients; CI, confidence interval.

based on the histogram of $\mathrm{HE}$ staining in the induction group were significantly higher than those of the control group. According to previous reports $(20,30,31)$, SD (or variance or coefficients of variation) and kurtosis derived from the histopathological image were positively associated with frequency and distribution of pixels, which was presumably related to tissue heterogeneity. Therefore, our histopathological results confirmed that a low dose of sorafenib could induce a more heterogeneous HCC compared with HCC without intervention.

Higher MK and lower $\alpha$ were shown to be associated with the complexity of various cancers, such as rectal and prostate cancers $(7,32)$. Significantly increased $M K$ and slightly decreased $\alpha$ were found within 24 hours after sorafenib treatment on a subcutaneous HCC model, which were caused by many intra- and intercellular factors including necrosis in the tumor (15). Jansen et al. also found that metastatic nodes in head and neck squamous cell carcinoma had higher heterogeneity when they were necrotic (33). In our study, increased MK and decreased $\alpha$ in the sorafenib induction group were correlated with SD and kurtosis, two histopathological markers reflecting the intratumoral heterogeneity of the tumor $(20,30)$. Meanwhile, they were also correlated with NF. As a result, the non-uniformly distributed necrosis within the tumor was presumably one of the most important causes of increased tumor heterogeneity when a low dose of sorafenib was used. However, no significant difference was found in tumor size between the two groups at our study time points. This implies the significance of functional parameters $\mathrm{MK}$ and $\alpha$ which changed earlier than the morphological index of HCC. Similar results were also shown after irradiation of nasopharyngeal carcinoma in a xenograft model (34). However, there were conflicting results regarding the changing trends of $M K$ and $\alpha$ in studies evaluating neoadjuvant chemoradiotherapy of rectal cancer
$(8,35)$. The discrepancy might be explained by complete tumor cell death, liquefaction, or massive necrosis, as well as therapy-induced fibrosis, which could reduce the tumor heterogeneity in these studies. To avoid complete or massive necrosis, which is likely when applied with a full dosage (4), we used a low dose of sorafenib to observe the tumor heterogeneity more closely at multiple time points.

In our study, MD and DDC exhibited significant increases after sorafenib treatment, which might be explained by decreased tumor cell density, increased intratumoral necrosis, and increased extracellular spaces. These values again showed positive correlations with histopathological SD, kurtosis, and NF, which indicate their potential to describe the complexity of tumor microstructure, particularly after treatment.

However, no correlations were found between DKI and SEM parameters, and MVD and Ki-67 metrics in the induction group. There were several possible explanations. First, DKI and SEM parameters might not have been sensitive to alterations in tumor cell proliferation and angiogenesis in our observation period. Second, tumor cell death and tissue necrosis might be the major contributing factors leading to the increased heterogeneity after sorafenib intervention (15). Third, the positioning of ROI which covered the necrotic areas might impair the demonstration of the real effects of cell proliferation and angiogenesis on these DKI and SEM parameters.

Differing from the sorafenib induction group, DKI and SEM parameters in the control group was not correlated with any histopathological markers. This might be due to the balance maintained between various tissue compositions in the natural course of HCC growth within our observational time points. Cell proliferation and tissue necrosis, which may have opposite effects on these DWI parameters, are two major counteracting factors leading 
to intratumoral complexity (4), and we speculate that the balance between them is broken by sorafenib.

Our study has several limitations. First, the measurement on the slice showing the greatest dimension of the tumor may not have adequately represented the heterogeneity of the whole tumor (36). Second, although great efforts were made to match the location of the tumors between DWI and histopathological images, no exact co-registration method was available, which may have led to some errors. Furthermore, until now, there has been no wellestablished histopathological standard for evaluation of tumor heterogeneity. However, with digital whole slide scanning technology and quantitative image analysis such as histogram, and grayscale values from HE staining representing different tissue components, their distribution, and even spatial interrelationships may be analyzed more reliably and objectively with gray-level co-occurrence matrix methods, such as entropy, contrast, and correlation $(22,23,37,38)$. Third, although hemorrhage was excluded from ROIs, micro-hemorrhage, which was invisible on MR images, may have influenced the accuracy of the DKI and SEM results. Fourth, the animal sample size was relatively small and might have led to a wider $95 \%$ CI for correlations between imaging parameters and histopathological results of HCC in the sorafenib induction group. Further studies with more animal models are warranted to verify our results before translation to clinical practice. Fifth, multiple time points might be a confounder for studying the correlations between the imaging and histopathological parameters. However, the two-way ANOVA test revealed no significant effects from these time points on DKI and SEM parameters. Last, this study focused on DKI and SEM parameters, which may only reflect some aspects of HCC heterogeneity. A recent report found that histogram analysis based on blood-oxygenation-level-dependent (BOLD) and dynamic contrast-enhanced (DCE)-MRI may provide a quantification of HCC heterogeneity as well (39). However, the latter techniques are time-consuming and more complicated.

In conclusion, DKI and SEM could reflect intratumoral heterogeneity of HCC and monitor its dynamic changes after application of low-dose sorafenib. Parameters of DKI and SEM may be potentially used for evaluating tumor heterogeneity of HCC.

\section{Acknowledgments}

Dr. Hua Lyu and Dr. Hualing Song from Shanghai
University of Traditional Chinese Medicine provided statistical advice in this study.

Funding: This research has received funding from the National Natural Science Foundation of China (81371542), Shanghai Health Committee (XBR2013115), and the Siming Scholar Foundation of Shuguang Hospital Affiliated to Shanghai University of Traditional Chinese Medicine.

\section{Footnote}

Conflicts of Interest: The authors have no conflicts of interest to declare.

Ethical Statement: The investigation was approved by the Institutional Animal Care and Use Committee of Zhongshan Hospital, Fudan University.

\section{References}

1. De Sousa E Melo F, Vermeulen L, Fessler E, Medema JP. Cancer heterogeneity--a multifaceted view. EMBO Rep 2013;14:686-95.

2. Greaves M. Evolutionary determinants of cancer. Cancer Discov 2015;5:806-20.

3. Burrell RA, Swanton C. Tumour heterogeneity and the evolution of polyclonal drug resistance. Mol Oncol 2014;8:1095-111.

4. Yang SH, Lin J, Lu F, Han ZH, Fu CX, Lv P, Liu H, Gao DM. Evaluation of antiangiogenic and antiproliferative effects of sorafenib by sequential histology and intravoxel incoherent motion diffusion-weighted imaging in an orthotopic hepatocellular carcinoma xenograft model. J Magn Reson Imaging 2017;45:270-80.

5. Bai Y, Lin Y, Tian J, Shi D, Cheng J, Haacke EM, Hong X, Ma B, Zhou J, Wang M. Grading of gliomas by using monoexponential, biexponential, and stretched exponential diffusion-weighted MR imaging and diffusion kurtosis MR imaging. Radiology 2016;278:496-504.

6. Jensen JH, Helpern JA. MRI quantification of nonGaussian water diffusion by kurtosis analysis. NMR Biomed 2010;23:698-710.

7. Liu X, Zhou L, Peng W, Wang H, Zhang Y. Comparison of stretched-exponential and monoexponential model diffusion-weighted imaging in prostate cancer and normal tissues. J Magn Reson Imaging 2015;42:1078-85.

8. Hu F, Tang W, Sun Y, Wan D, Cai S, Zhang Z, Grimm $\mathrm{R}$, Yan X, Fu C, Tong T, Peng W. The value of diffusion kurtosis imaging in assessing pathological complete 
response to neoadjuvant chemoradiation therapy in rectal cancer: a comparison with conventional diffusion-weighted imaging. Oncotarget 2017;8:75597-606.

9. Orton MR, Messiou C, Collins D, Morgan VA, Tessier J, Young H, deSouza N, Leach MO. Diffusion-weighted MR imaging of metastatic abdominal and pelvic tumours is sensitive to early changes induced by a VEGF inhibitor using alternative diffusion attenuation models. Eur Radiol 2016;26:1412-9.

10. Wang Y, Hu D, Yu H, Shen Y, Tang H, Kamel IR, Li Z. Comparison of the diagnostic value of monoexponential, biexponential, and stretched exponential diffusionweighted MRI in differentiating tumor stage and histological grade of bladder cancer. Acad Radiol 2019;26:239-46.

11. Caldwell S, Park SH. The epidemiology of hepatocellular cancer: from the perspectives of public health problem to tumor biology. J Gastroenterol 2009;44:96-101.

12. Tang ZY, Ye SL, Liu YK, Qin LX, Sun HC, Ye QH, Wang L, Zhou J, Qiu SJ, Li Y, Ji XN, Liu H, Xia JL, Wu ZQ, Fan J, Ma ZC, Zhou XD, Lin ZY, Liu KD. A decade's studies on metastasis of hepatocellular carcinoma. J Cancer Res Clin Oncol 2004;130:187-96.

13. Rosenkrantz AB, Sigmund EE, Winnick A, Niver BE, Spieler B, Morgan GR, Hajdu CH. Assessment of hepatocellular carcinoma using apparent diffusion coefficient and diffusion kurtosis indices: preliminary experience in fresh liver explants. Magn Reson Imaging 2012;30:1534-40.

14. Goshima S, Kanematsu M, Noda Y, Kondo H, Watanabe $\mathrm{H}$, Bae KT. Diffusion kurtosis imaging to assess response to treatment in hypervascular hepatocellular carcinoma. AJR Am J Roentgenol 2015;204:W543-9.

15. Chen X, Ma Z, Huang Y, He L, Liang C, Shi C, Zhang Z, Liang C, Liu Z. Multiparametric MR diffusion-weighted imaging for monitoring the ultra-early treatment effect of sorafenib in human hepatocellular carcinoma xenografts. J Magn Reson Imaging 2017;46:248-56.

16. Liu L, Cao Y, Chen C, Zhang X, McNabola A, Wilkie D, Wilhelm S, Lynch M, Carter C. Sorafenib blocks the RAF/ MEK/ERK pathway, inhibits tumor angiogenesis, and induces tumor cell apoptosis in hepatocellular carcinoma model PLC/PRF/5. Cancer Res 2006;66:11851-8.

17. Sun K, Chen X, Chai W, Fei X, Fu C, Yan X, Zhan Y, Chen K, Shen K, Yan F. Breast cancer: diffusion kurtosis MR imaging-diagnostic accuracy and correlation with clinical-pathologic factors. Radiology 2015;277:46-55.

18. Li RK, Zeng MS, Rao SX, Qiang JW, Dai YM, Ji Y, Chen
CZ, Renate J. Using a 2D multibreath-hold susceptibilityweighted imaging to visualize intratumoral hemorrhage of hepatocellular carcinoma at 3T MRI: correlation with pathology. J Magn Reson Imaging 2012;36:900-6.

19. Natarajan S, Juneja M, Pallam NK, Boaz K, Mohindra A, Lewis A. A novel technique to assess chromatin texture using pixel optical densitometry in oral squamous cell carcinoma. Microsc Res Tech 2012;75:1119-23.

20. Sadi MV, Barrack ER. Image analysis of androgen receptor immunostaining in metastatic prostate cancer. Heterogeneity as a predictor of response to hormonal therapy. Cancer 1993;71:2574-80.

21. Lu C, Ji M, Ma Z, Mandal M. Automated image analysis of nuclear atypia in high-power field histopathological image. J Microsc 2015;258:233-40.

22. Xu Y, Jiao L, Wang S, Wei J, Fan Y, Lai M, Chang EI. Multi-label classification for colon cancer using histopathological images. Microsc Res Tech 2013;76:1266-77.

23. Belhomme P, Toralba S, Plancoulaine B, Oger M, Gurcan $\mathrm{MN}$, Bor-Angelier C. Heterogeneity assessment of histological tissue sections in whole slide images. Comput Med Imaging Graph 2015;42:51-5.

24. Geng W, Ng KT, Sun CK, Yau WL, Liu XB, Cheng Q, Poon RT, Lo CM, Man K, Fan ST. The role of proline rich tyrosine kinase 2 (Pyk2) on cisplatin resistance in hepatocellular carcinoma. PLoS One 2011;6:e27362.

25. Huang Y, Lin Y, Hu W, Ma C, Lin W, Wang Z, Liang J, Ye W, Zhao J, Wu R. Diffusion kurtosis at 3.0T as an in vivo imaging marker for breast cancer characterization: correlation with prognostic factors. J Magn Reson Imaging 2019,49:845-56.

26. Landis JR, Koch GG. The measurement of observer agreement for categorical data. Biometrics 1977;33:159-74.

27. Nelson DA, Tan TT, Rabson AB, Anderson D, Degenhardt K, White E. Hypoxia and defective apoptosis drive genomic instability and tumorigenesis. Genes Dev 2004;18:2095-107.

28. Kwee TC, Galban CJ, Tsien C, Junck L, Sundgren PC, Ivancevic MK, Johnson TD, Meyer CR, Rehemtulla A, Ross BD, Chenevert TL. Intravoxel water diffusion heterogeneity imaging of human high-grade gliomas. NMR Biomed 2010;23:179-87.

29. Yang Z, Tang LH, Klimstra DS. Effect of tumor heterogeneity on the assessment of Ki67 labeling index in well-differentiated neuroendocrine tumors metastatic to the liver: implications for prognostic stratification. Am J 
Surg Pathol 2011;35:853-60.

30. Lucero HA, Patterson S, Matusuura S, Ravid K. Quantitative histological image analyses of reticulin fibers in a myelofibrotic mouse. J Biol Methods 2016;3:e60.

31. Dang Y, Lan X, Zhang Q, Li L, Huang Y. Analysis of grayscale characteristics in images of labeled microtubules from cultured cardiac myocytes. Microsc Microanal 2015;21:334-42.

32. Zhu L, Pan Z, Ma Q, Yang W, Shi H, Fu C, Yan X, Du L, Yan F, Zhang H. Diffusion kurtosis imaging study of rectal adenocarcinoma associated with histopathologic prognostic factors: preliminary findings. Radiology 2017;284:66-76.

33. Jansen JF, Schoder H, Lee NY, Stambuk HE, Wang Y, Fury MG, Patel SG, Pfister DG, Shah JP, Koutcher JA, Shukla-Dave A. Tumor metabolism and perfusion in head and neck squamous cell carcinoma: pretreatment multimodality imaging with $1 \mathrm{H}$ magnetic resonance spectroscopy, dynamic contrast-enhanced MRI, and [18F] FDG-PET. Int J Radiat Oncol Biol Phys 2012;82:299-307.

34. Zheng X, Chen Y, Xiao Y, Zheng D, Chen W. Early diagnosis of radio-insensitive human nasopharyngeal carcinoma xenograft models by diffusion kurtosis imaging. Magn Reson Imaging 2019;55:128-32.

35. Zhu HB, Zhang XY, Zhou XH, Li XT, Liu YL, Wang S, Sun YS. Assessment of pathological complete response

Cite this article as: Guo R, Yang $\mathrm{SH}$, Lu F, Han ZH, Yan $\mathrm{X}$, Fu CX, Zhao ML, Lin J. Evaluation of intratumoral heterogeneity by using diffusion kurtosis imaging and stretched exponential diffusion-weighted imaging in an orthotopic hepatocellular carcinoma xenograft model. Quant Imaging Med Surg 2019;9(9):1566-1578. doi: 10.21037/qims.2019.08.18 to preoperative chemoradiotherapy by means of multiple mathematical models of diffusion-weighted MRI in locally advanced rectal cancer: a prospective single-center study. J Magn Reson Imaging 2017;46:175-83.

36. Wei Y, Gao F, Wang M, Huang Z, Tang H, Li J, Wang Y, Zhang T, Wei X, Zheng D, Song B. Intravoxel incoherent motion diffusion-weighted imaging for assessment of histologic grade of hepatocellular carcinoma: comparison of three methods for positioning region of interest. Eur Radiol 2019;29:535-44.

37. Liu Y, Xu X, Yin L, Zhang X, Li L, Lu H. Relationship between glioblastoma heterogeneity and survival time: an MR imaging texture analysis. AJNR Am J Neuroradiol 2017;38:1695-701.

38. Liu L, Liu Y, Xu L, Li Z, Lv H, Dong N, Li W, Yang Z, Wang Z, Jin E. Application of texture analysis based on apparent diffusion coefficient maps in discriminating different stages of rectal cancer. J Magn Reson Imaging 2017;45:1798-808.

39. Hectors SJ, Wagner M, Bane O, Besa C, Lewis S, Remark R, Chen N, Fiel MI, Zhu H, Gnjatic S, Merad M, Hoshida Y, Taouli B. Quantification of hepatocellular carcinoma heterogeneity with multiparametric magnetic resonance imaging. Sci Rep 2017;7:2452. 